\title{
Una revisión sociotécnica de las redes sociales de una organización universitaria
}

\author{
Marco Brandão*
}

Artículo recibido:

20 de mayo de 2020

Artículo aceptado:

18 de septiembre de 2020

Artículo de revisión

\section{Resumen}

En ese artículo se plantean los indicadores de eficiencia para las redes sociales (RS) de una organización universitaria y se proponen algunas bases teórico-prácticas para investigaciones futuras que consideren importante el creciente uso de las RS en procesos de gestión. Se trata de una revisión teórico-conceptual sobre los aspectos sociotécnicos de las RS capaces de subsidiar una metodología de evaluación. Esta investigación es de naturaleza y enfoque aplicado, con objetivos exploratorios y descriptivos, realizando un análisis sociotécnico de las RS, el estudio de los lineamientos de la organización universitaria para las RS, el análisis del flujo de la

* Departamento de Fundamentos de Ciencias de la Sociedad, Universidad Federal Fluminense (UFF, Brasil) marcobrandao@zipmail.com.br 
información y las proposiciones de los indicadores de eficiencia que serán mencionados. Este artículo es producto parcial del proyecto principal denominado "El flujo de información a través de las redes sociales", que plantea como principal producto una metodología de evaluación de eficiencia de las RS de una organización universitaria.

Palabras clave: Redes Sociales; Análisis Sociotécnico; Flujo de Información; Indicadores de Eficiencia

\section{A socio-technical review of Social Media in a univer- sity organization \\ Marco Brandão}

\section{Abstract}

This paper intends to define efficiency indicators for using Social Media (SM) in a university setting, as well as to provide theoretical-practical bases for future research that takes into consideration the increasing use of SM in management processes. It is a theoreticalconceptual review of the socio-technical aspects of SM that is capable of subsidizing an evaluation methodology. It engages an applied approach, with exploratory and descriptive objectives, performing as such, a socio-technical analysis of SM, a study of university organizational guidelines for SM, an information flow analysis, and a proposal for efficiency indicators. This paper is part of a larger project entitled, "Information Flow through Social Media" which proposes as its main outcome an efficiency evaluation methodology for SM within university organizations.

Keywords: Social Media; Socio-Technical Analysis; Information Flow; Efficiency Indicators 


\section{INTRODUCCIÓN}

T a información en la web asume muchas posibilidades de usos y de inter-

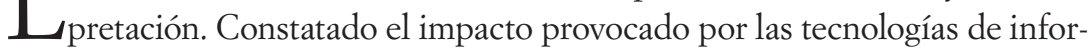
mación y comunicación (TIC), sus aspectos técnicos han cambiado la manera de actuación de organizaciones y personas a partir de las características que tiene la información en el ciberespacio, sus potencialidades y sus recursos.

Uno de estos recursos son las redes sociales (RS). Celaya (2008: 123) define las RS como "lugares en Internet donde las personas publican y comparten todo tipo de información, personal y profesional, con terceras personas, conocidos y absolutos desconocidos", y la Wikipedia como "una estructura social que se puede representar en forma de uno o varios grafos donde los nodos representan individuos y las aristas las relaciones entre ellos".

Estos "lugares" y la "estructura social" se presentan en la forma de sitios y/o aplicaciones para dispositivos móviles que operan en niveles diversos en la web, donde las personas y las organizaciones que los componen se conectan a partir de intereses o valores comunes. De esa manera, se pueden identificar las relaciones entre individuos, empresas, gobiernos, temas, permitiendo el flujo de información y su uso estratégico y eficiente.

El uso de las RS es tendencia en todo el mundo, como lo afirma Contreras Orozco (2017: 228): "hoy en día se han convertido en un espacio con muchas oportunidades para cualquier ámbito de actividad productiva, y pueden ser un instrumento fundamental para construir nuevas relaciones de cercanía y participación social en las actividades gubernamentales". Además, desde que fueron creadas hasta la fecha, las RS han roto las barreras en cuanto a los procesos de comunicación e internacionalización, debido a que su alcance está más allá de las fronteras territoriales, culturales e ideológicas, en relación con los flujos de información internacional (Sánchez Vanderkast, 2002: 58). Por ello es que están llamadas a ser los medios líderes como canales de comunicación.

Asumiendo esa tendencia, en 2019 se consideró a México como el tercer país del globo en uso de las RS, lo que según el estudio The Global State of Digital in 2019 de la plataforma de gestión de RS Hootsuite (Kemp, 2019) significa que ha llegado a los 83 millones de usuarios. Entre otros, eso implica que las RS han impactado en la vida de más de la mitad de la población del país. Nos preguntamos: ¿de qué forma?

Son distintos los intereses que unen a las personas alrededor de valores, temas, organizaciones, así como son distintos los objetivos que tienen las diferentes RS. Caldevilla Dominguez (2010) clasifica en cinco los tipos de RS de acuerdo a la prioridad de sus usos: 1) Generales (e.g. Facebook, Twitter); 2) 
Fans - para compartir intereses en común (e.g.: películas, música, deportes, etc.); 3) Estados vitales - grupos específicos de personas (e.g.: padres, personas que están por casarse, abuelos, etc.); 4) Contenidos - para compartir videos y/o fotografías (e.g.: YouTube, Instagram), y 5) Profesionales y activistas (e.g.: LinkedIn, Academia.edu, etc.). Autores como Celaya (2008: 123) dividen a las RS en 1) Profesionales, 2) Generalistas y 3) Especializadas. En ambos casos, los autores nos dan a entender que las RS se distinguen en cuanto a los objetivos y, por lo tanto, sus seguidores las prefieren por sus necesidades de información.

Con base en el estudio de Hootsuite (Kemp, 2019), las RS más conocidas y utilizadas en México son, en primer lugar, YouTube, con $95 \%$ de usuarios activos; en segundo lugar Facebook, con $93 \%$ de usuarios activos; en tercer lugar WhatsApp, con 87 \% de usuarios activos; en cuarto lugar FB Messenger, con $64 \%$ de usuarios activos, y en quinto lugar Twitter, con $57 \%$ de usuarios activos, acompañando la tendencia mundial de uso de RS de carácter general.

Con ese gran uso de RS generales por la población mexicana, la evaluación de su impacto es difícil y compleja debido a los diferentes usos que uno puede hacer con las RS. Es decir, las RS pueden servir para todo, desde la comunicación, entretenimiento, movilidad, hasta las actividades laborales, comerciales, gubernamentales, académicas: en ese universo, seguro habrá personas que pertenecen a distintos grupos, temas, guardando con ellos distintas relaciones y objetivos.

Por esa razón, los estudios sobre el impacto de las RS deben relacionarse tanto con sus objetivos en cuanto red como con las características e intereses de los usuarios que los hacen participar o seguir a grupos, temas u organizaciones. Existen indicadores que han sido desarrollados para analizar cómo es el flujo de la información en las RS dependiendo de esas relaciones, actividades y objetivos que persiga el individuo al relacionarse en el ciberespacio a través de las RS.

Por otro lado, las organizaciones que ocupan las RS esperan que ellas ofrezcan algún impacto a sus objetivos, a sus negocios, por lo que solamente el reconocimiento y la visibilidad de su marca no serían suficientes ante su comunidad de seguidores: se espera alguna influencia en ellos para que se identifiquen, se posicionen, consuman o se informen sobre los productos y los servicios de estas organizaciones.

Estos aspectos sociotécnicos de las RS pueden proveer a las organizaciones de información privilegiada sobre sus actividades a partir de una comunidad especializada de usuarios ubicados por el interés en sus acciones; sin embargo, en el universo de las RS generales, ¿de qué forma se puede considerar que estos aspectos impactan en los objetivos de la organización? 
El propósito de este artículo es diseñar, a partir de los aspectos sociotécnicos encontrados en las RS y en las organizaciones, los posibles indicadores de eficiencia para las RS utilizadas por una organización y proponer bases teórico-prácticas para los estudios de la información teniendo así la posibilidad de revisar en futuros trabajos relacionados a las RS.

\section{UNIVERSO Y METODOLOGÍA DE ESTUDIO}

Frente a la diversidad de las relaciones y objetivos que establecen las organizaciones y los individuos con las RS, el universo contemplado en ese estudio son las RS de una entidad académica de la Universidad Nacional Autónoma de México (UNAM): el Instituto de Investigaciones Bibliotecológicas y de la Información (IIBI), el cual ofrece el espacio ideal para el tema al ser una de sus especialidades.

El IIBI ha cumplido en 2019 una década en haber puesto en funcionamiento sus RS y con ello el haber cambiado y ajustado su forma de comunicación a las nuevas tendencias de comunicación social, tanto con su comunidad interna como con la comunidad universitaria nacional e internacional; ha sido una de las entidades académicas de la UNAM pioneras en el uso de la RS, habiendo mostrado que las RS serían y serán los medios de comunicación del siglo XXI. De acuerdo con lo anterior, es oportuno indagar la manera en que las RS del IIBI han pasado de ser sólo un punto de encuentro a canales de comunicación que cumplen con las funciones sustantivas de investigación, docencia y difusión de la cultura como organización universitaria, objetivos de la UNAM. Actualmente cuenta con más de 34000 usuarios en sus perfiles de Facebook, Twitter y YouTube, un número expresivo dentro de las tendencias apuntadas por Hootsuite (Kemp, 2019).

Además, el uso de las RS en la educación superior ha llamado la atención de muchos investigadores que buscan entenderlas con las finalidades educativas, que según Veletsianos (2011: 1) incluyen una gran variedad de tópicos:

(...) youth's online practices (Ito et al. 2009), the relationship between online social networks and educational outcomes (Kirschner \& Karpinski 2010; Junco et al. 2011), and students' attitudes towards online social networks (Hew 2011). Recent literature has also proposed that participatory Internet technologies may offer expanded opportunities to scholars for professional endeavours, transforming the ways academics engage in teaching and research (Greenhow et al. 2009; Katz 2010). For example, scholars can maintain ongoing interactions with geographically dispersed colleagues, post drafts of their manuscripts online and invite colleagues to comment and critique their work prior to formal publication 
(e.g. Conole 2011), or they can work with others on a large scale, capitalizing on a group's collective intelligence and division of labour, to solve hard problems [e.g. see Gowers \& Nielsen (2009) and Gowers success in engaging numerous individuals in finding a proof for a complex mathematical problem].

En relación con las maneras con las cuales la educación y la investigación han sido desarrolladas por estas organizaciones, las RS han posibilitado prácticas que permiten desde la innovación de los procesos de instrucción (enseñanza y aprendizaje) hasta la gestión de la organización para la percepción de sus resultados y de su impacto. Incluso, apunta Veletsianos (2011: 9), igual han sido ocupadas como factor de identidad corporativa y profesional de usuarios que ponen en evidencia sus trabajos y éxitos profesionales:

- Mention of my presentation at [University Name]: [URL]

- Heading to [University Name] to give a talk to the [name] Group: [URL]

- Appreciative for the comments and the hundreds of views/day on this presentation: [URL].

In addition to sharing information regarding lectures and presentations, participants also shared links to interviews that they gave:

- In my interview with the editor of [name], I discuss [topic]: [URL]

- [URL]: My interview on [topic related to professional interests]

- Interview with [Periodical name]: [URL]

Enfocada, por lo tanto, en el aspecto de la gestión de la información de una organización universitaria, esta investigación tuvo naturaleza y enfoque aplicado, con objetivos exploratorios y descriptivos. Como un estudio exploratorio (Triviños, 1987: 109-110), se hizo una revisión bibliográfica y documental para identificar las características de la organización y los indicadores posibles para sus RS -el objetivo de ese artículo-y, a continuación, proponer una metodología de evaluación de eficiencia con estos indicadores.

Por ello, se realizaron las acciones siguientes: 1) un análisis sociotécnico de la organización universitaria y de las RS en conformidad con los abordajes de Trist y Bamforth (1951), Emery y Trist (1960), Manrique Valenzuela y Gonzalez Álvarez (2013), Caldevilla Dominguez (2010), Contreras Orozco (2017), Hütt Herrera (2012), revisando sus estructuras y realidad actual; 2) el estudio de los lineamientos de la UNAM para las RS (UNAM, 2014);3) el análisis del flujo de la información con base en Devlin (1991) y Sánchez Vanderkast (2002); y 3) la proposición de los indicadores de eficiencia que pueden ser mencionados. 
Ese artículo fundamenta el proyecto de investigación "El flujo de información a través de las redes sociales", que plantea como principal producto una metodología de evaluación de eficiencia de las RS de organizaciones universitarias, llevado a cabo en el marco de una estancia de investigación en el IIBI, la cual fue auspiciada por el Gobierno de México a través de la Agencia Mexicana de Cooperación Internacional para el Desarrollo (AMEXCID).

\section{UNA REVISIÓN SOCIOTÉCNICA DE LAS RS}

Trist y Bamforth (1951) y Emery y Trist (1960) fueron los primeros que acuñaron la expresión socio-technical system en un estudio sobre organizaciones. Para ellos, un sistema sociotécnico involucra los aspectos sociales de las personas, la colectividad, los aspectos técnicos de la estructura organizacional y los procesos. Un sistema sociotécnico debe describir una compleja interacción entre los seres humanos, las máquinas y el ambiente donde se encuentran.

Los sistemas sociotécnicos tienen la premisa de que la cultura y la tecnología son variables dependientes, es decir, el cambio en una produce cambios en la otra. Por eso, su abordaje ha sido empleado en muchos estudios, entre ellos los arqueológicos y económicos, y actualizado por investigadores como Perrow (1978), Ropohl (1982), Cummings y Worley (1993), Badham, Clegg y Wall (2000) y Geels (2004) (citados por Manrique Valenzuela y Gonzalez Álvarez, 2013:2).

Kingdon (1973:3) establece que un enfoque del sistema sociotécnico está constituido de tres subsistemas principales: el sistema gerencial, responsable del desarrollo organizacional y la toma de decisiones; el sistema social, soporte para el flujo de información requerido por el sistema técnico, y el sistema técnico, que garantiza el flujo de la información.

En rigor, por lo tanto, las RS requieren un abordaje sociotécnico. En el universo de ese estudio, este abordaje exige una mirada sobre la Organización UNAM a través de su Plan de Desarrollo Institucional (el sistema gerencial), sobre la comunidad universitaria (el sistema social) y sobre sus RS (el sistema técnico).

\section{La UNAM cómo sistema gerencial}

La UNAM es una de las más prestigiadas y reconocidas instituciones educativas y científicas del mundo. En su último Plan de Desarrollo Institucional ha elegido como uno de sus programas estratégicos el acceso, uso, aplicación y desarrollo de las TIC y de las TAC (Tecnologías del Aprendizaje y el Conocimiento) 
para la mejora del ejercicio y cumplimiento óptimo de las funciones de la Universidad (UNAM, 2017: Programa 7).

Dicho Programa Estratégico influyó en las decisiones respecto de las RS de la UNAM como parte del sistema técnico que garantiza el flujo de la información, reconociendo que

La Universidad del siglo XXI debe concebirse como un espacio en el que las TIC impacten en todos sus ámbitos. La modernización de estos procesos deberá incluir la forma en que se enseña y en la que aprenden los alumnos, la estructura y funcionamiento de las bibliotecas, la edición y publicación de libros y revistas, la integración de acervos institucionales, la evaluación permanente de acciones, la difusión de las actividades culturales, la administración, las gestiones jurídicas y la comunicación entre universitarios. (UNAM, 2017:30)

Eso implicó la creación de un plan maestro para el uso y aplicación de las TIC en todos los ámbitos para la sistematización de la información relacionada con la Universidad y su quehacer, así como para la interacción con las comunidades universitaria y externa. De ahí la creación del Instructivo de uso para la Redes Sociales Institucionales de la UNAM (UNAM, 2014), un documento que establece que las entidades y dependencias incorporen buenas prácticas en el uso de las RS Twitter, Facebook y YouTube, así como la conformación del sitio web institucional, en el que se deben colocar, entre otros elementos, los logos de la UNAM, la entidad o dependencia, la misión, el directorio, etc., con el objetivo principal de integrar las cuentas de la RS institucionales para enriquecer la interacción y la colaboración con la comunidad universitaria en un trabajo conjunto de sus entidades y dependencias para una mayor visibilidad y apreciación del quehacer universitario (UNAM, 2014:3).

Antes de esa política de información de la UNAM, el IIBI había creado sus RS institucionales, siendo una entidad pionera en la Universidad. A finales de 2008, cuando aún era el Centro Universitario de Investigaciones Bibliotecológicas (CUIB), se decidió crear un perfil con atributo de persona en la RS Facebook, migrando esta cuenta a una página de fans a inicios de 2010 y de ahí, en 2012, con los cambios permitidos por la plataforma y tras el cambio del CUIB para el IIBI, para un perfil institucional, impactando en el alcance de seguidores de la entidad.

Acompañando ya los lineamentos del PDI 2011-2015 (UNAM, 2012), el IIBI incorpora a su Plan de Desarrollo 2012-2016 (Ríos Ortega, 2012) la línea de acción $n^{\circ} 3$, la cual tiene entre sus consideraciones "promover los productos de investigación por medio de las redes académicas y sociales del IIBI; intensificar la transmisión en vivo utilizando los canales de la web: Ustream y Livestream; 
asimismo extender el acceso abierto en YouTube y en el repositorio de videos del Instituto" (Ríos Ortega, 2012: 22-23).

El objetivo era aumentar la visibilidad de los productos de investigación y de las actividades académicas organizadas por el IIBI, además de concluir la incorporación en acceso abierto de la producción editorial del Instituto, cumpliendo con las enmiendas 14 y 16 de dicho PDI 2011-2015 (UNAM, 2012), el cual ya reportaba el uso de las RS como medio de comunicación con la comunidad universitaria y al refuerzo de la visibilidad en internet de la Universidad.

Así, en esa misma ocasión, al Instituto se han incorporado plenamente las RS Facebook, Twitter y YouTube, ampliando su visibilidad y presencia en Iberoamérica, fortaleciendo la comunicación entre pares de otras partes del mundo e incrementado la vinculación interinstitucional, potenciando la consulta y acceso a los productos de investigación y difusión. "Estas redes han sido muy importantes para promover y favorecer el acceso abierto de las publicaciones del Instituto y a los eventos académicos que realiza”, concluye el $2^{\circ}$ Informe de Actividades 2013-2014 del IIBI (Ríos Ortega, 2014: 11).

Por lo anterior, se constata que, en los últimos años, el sistema gerencial que comprende la UNAM ha ido considerando las RS para su desarrollo organizacional y el IIBI ha adoptado prácticas basadas en esa tecnología (el sistema técnico).

\section{La UNAM como sistema social}

La UNAM como sistema social cuenta con el soporte para procurar el flujo de la información entre su público universitario (estudiantes, docentes, investigadores, técnicos) y el público externo. Estos públicos se relacionan de formas distintas con la Universidad y las informaciones que ellos necesitan o por ellos proporcionadas sirven a diferentes propósitos. Sin embargo, queda claro que las actividades científicas y educativas de la UNAM basan sus cimientos en la generación, transmisión, divulgación y aplicación del saber de sus investigadores, docentes y funcionarios: son ellos quienes realizan los aportes que contribuyen con el desarrollo social, económico, científico, cultural y político de México, cómo registra su PDI (UNAM, 2017: 11).

Las RS de la UNAM, por lo tanto, en una primera mirada, son productos del quehacer universitario dirigidos hacia los estudiantes, docentes, investigadores, técnicos de la institución y hacia al público externo, el cual se conforma también de estudiantes, investigadores y docentes de otras instituciones nacionales e internacionales y la sociedad en general (ciudadanos, organizaciones, gobierno, etcétera). De hecho, en ese último rubro se encuentran los 83 
millones de mexicanos que hacen del país el tercero del mundo en uso de RS (Kemp, 2019). La respuesta de estos distintos públicos de las RS a los servicios y productos de la Universidad son muy variables y expresan las diferentes relaciones y objetivos que establecen los individuos con sus RS.

Entre las conclusiones del Estudio sobre los Hábitos de los Usuarios de Internet en México 2019 (AIMX, 2019), de la Asociación de Internet Mx, encontramos que las RS son la principal actividad en línea de los mexicanos para utilidad en materia de comunicación, siendo Facebook la principal RS, mientras que $31 \%$ de los usuarios pasan su tiempo conectados en alguna RS. En ese universo se encuentran las entidades académicas de la UNAM y sus usuarios moviéndose entre productores y prosumidores de la información (Terra y Rodríguez García, 2013: 1298). En ese momento se establece el reto para los profesionales de la información que cumplen con la misión de organizar, sistematizar, disponer y atender la comunidad universitaria de la UNAM en sus necesidades informativas.

Torres Vargas et al. (2015: 2-3) considera que la innovación tecnológica ha generado una ruptura que hace desaparecer productos y servicios y ocasiona el cambio de las organizaciones, introduciendo nuevas estructuras, funciones y responsabilidades internas y externas, redefiniendo las estrategias que la influencian, y eso ha impactado en las bibliotecas y servicios de información, tan necesarios al contexto universitario.

Así, en la reestructuración técnica del profesional de la información, se han contemplado cambios significativos de su actuación debido a las transformaciones tecnológicas y a la forma en cómo la producción, la organización, el acceso y el uso de la información se procesa con ellos. En ese sentido, en un sistema social como el de la UNAM, las implicaciones son extremamente significativas, ya sea por el contexto mexicano, ya sea por la naturaleza de la institución de generar, transmitir, divulgar y aplicar el saber de sus investigadores, docentes y funcionarios a un público diversificado y con diferentes necesidades de información.

Si antes la biblioteca era considerada como el espacio de información donde las personas podrían satisfacer sus necesidades y objetivos de información, hoy en día ya no es exclusivo. Además de ampliarse en términos de su alcance, permitiéndose hacer disponible su acervo en línea, accesible a cualquier hora y en cualquier parte del mundo, la biblioteca trata en su cotidiano con otros espacios de información, con nuevas formas de producción y de consumo de información, con una nueva dinámica de la información, con ciclos cada vez más rápidos y afectos a usuarios que asumen sus diferentes papeles a través de ello: alguien que busca, pero que también ofrece información. 
Así también, como un sistema social, la UNAM alcanza a un público no restricto a sus estudiantes, docentes, investigadores y técnicos, ni solamente a un público externo ubicado cerca de la institución, sino también a un público de estudiantes, investigadores y docentes de instituciones internacionales, la sociedad de otros países (ciudadanos, empresas, gobierno, etc.) por medio de las TIC. La responsabilidad de sus profesionales -especialmente los profesionales de la información- está en brindar a México y al mundo el quehacer de la Universidad con recursos como las RS institucionales en un universo de miles de usuarios con miles de necesidades informativas.

\section{La UNAM cómo sistema técnico}

Por lo anterior, la Universidad reconoce las RS como uno de "los medios digitales más utilizados en la actualidad por la sociedad en general" y "las emplea como un medio para difundir el quehacer universitario que se gesta día a día" (UNAM, 2020). El Instructivo de uso para la Redes Sociales Institucionales de la UNAM deja claro que las RS son "un canal valioso de comunicación adicional" y "abren una posibilidad excepcional para un contacto inmediato e interacción con la comunidad universitaria y la sociedad" (UNAM, 2014: 3). Así que las RS forman parte del sistema técnico de la UNAM, siendo además parte de sus estrategias de relacionamiento, oferta de productos y servicios.

En el IIBI, la Secretaría Técnica es el área encargada de tres de los departamentos que trabajan de manera colaborativa para lograr un óptimo desarrollo de las actividades del Instituto relacionadas con su Plan de Desarrollo: el Departamento de Cómputo, el Departamento de Publicaciones y el Departamento de Difusión y Educación Continua. Entre sus tareas estratégicas se encuentran la divulgación de la actividad académica, de la producción literaria y de los servicios que presta el Instituto. El Departamento de Difusión y Educación Continua es el encargado de gestionar las RS de la entidad.

La actividad del IIBI en los medios electrónicos es indispensable para el desempeño de sus actividades e impacto social y ellos han sido seleccionados de acuerdo a la necesidad de comunicación y las tendencias actuales de la interacción social a través de internet, particularmente en el contexto mexicano. Desde las herramientas más básicas, como el correo electrónico, hasta las avanzadas como el streaming, esa diversidad de TIC que utiliza el Departamento de Difusión y Educación Continua de la entidad para llevar a cabo la información y la comunicación se debe a que el público al que se dirige está compuesto por grupos de personas de segmentos distintos entre sí y debido a esto sus necesidades de información, su manera de interactuar o simplemente 
recibir un mensaje varían de acuerdo a la edad, ubicación geográfica, condición económica, preparación académica, acceso a la tecnología, etc. Por este motivo, la comunicación que ocurre puede darse por un solo medio o por una combinación de todos los que dispone el Instituto. En el enfoque aquí propuesto referente a las RS, el sistema técnico considerado serán las cuentas oficiales de las RS:

$$
\begin{gathered}
\text { Facebook (https://www.facebook.com/IIBI.UNAM.MX) } \\
\text { Twitter (https://twitter.com/IIBI_UNAM_MX) } \\
\text { YouTube (https://www.youtube.com/user/CUIB100) }
\end{gathered}
$$

\title{
EL FLUJO DE INFORMACIÓN EN LAS RS: UN RETO PARA LOS PROFESIONALES DE LA INFORMACIÓN
}

\begin{abstract}
Torres Vargas et al. (2015: 4) apuntan que la gestión de las RS es también una atribución de los profesionales de la información en el nuevo contexto de producción, organización, acceso y uso de la información. De hecho, estos autores nos presentan como principales retos de dicho profesional en relación a las RS:
\end{abstract}

1) Plan and identify users and needs: Project the supply of information resources, staff training, and users guidance. Spread the news about the benefits of creating social networks for the community's information and knowledge. Establish guidelines, standards and quality indicators.

[...]

3) Implement the integration of social networks, applications and Web 2.0 tools in their institution website: These instruments are useful for the promotion of library services through platforms and in the organization and exchange of contents (YouTube, iTunes, Twitter, Facebook). (Torres Vargas et al., 2015: 7)

Además, identifican un tipo de usuario de información -los Web 2.0 usersa quienes les gusta la colaboración, el trabajo en equipo y las RS, lo que ha representado un enorme desafío para el nuevo bibliotecario, a quien llaman Blended Librarian (Torres Vargas et al., 2015: 7).

Además de estos retos, está la realidad de México: el tercer país en uso de las RS de carácter general, figurando Facebook, YouTube y Twitter entre las primeras, lo que abarca un público diversificado consumidor y prosumidor de información del que forma parte la comunidad universitaria, como lo confirma Veletsianos (2011: 10): "the findings indicate that scholars networked 
participation is a complex and multifaceted human activity where personal and professional identities blend, and where participatory digital practices meet individual reflections, fragmented updates, and social interaction".

En torno a esto es necesario alcanzar al usuario de las RS universitarias en sus necesidades de información, organizando, apartando, clasificando sus diferentes usos y buscando entender de qué forma estos aspectos impactan con los objetivos de la organización en el cumplimiento de sus programas estratégicos. Visto que los usuarios de las RS de carácter general tienen distintos objetivos y características, debe considerarse que quienes se vinculan a las RS de una organización universitaria tengan/tienen con ella alguna relación, ya sea como estudiantes, docentes, investigadores, funcionarios o profesionales del campo, externos o no a la organización.

Es bueno distinguir también que una RS de una organización universitaria no es lo mismo que una RS académica. Mientras que la segunda está constituida por redes especializadas (profesionales, colegios invisibles), la primera se trata de las redes institucionales de las que dispone la institución en estos canales. En estas RS, el flujo de la información sirve a distintos usuarios, circulando entre distintas personas, sectores y organizaciones. El matemático Keith Devlin (1991), en su libro Logic and Information, señala que la información abstracta sirve para clasificar estados de cosas concretos y el flujo de información para indicar la clasificación de esa información. Desde ese punto de vista, la información de las RS nos presenta un primer estado de cosas que transporta información acerca de un segundo estado de cosas: "la información de la información" (e.g.: la cantidad de seguidores en una RS, su tema/ campo de inserción, etc. son las informaciones; quiénes son estos seguidores, qué hacen, qué necesitan etc. forma parte del flujo de información).

Sánchez Vanderkast (2002: 56-57) ubica los estudios de flujos de información en conformidad con lo propuesto por David Bender: como un tópico de las políticas de información. Lo anterior, considerando los pronunciamientos principales de la automatización y de la era de la información que dominaron los años setenta y ochenta, se refiere a los flujos de información transfronteras, los cuales tiene sus fundamentos en los sistemas de cómputo, en las telecomunicaciones y en los contenidos, considerado los contenidos como 1) los servicios de datos disponibles por medio de bancos de datos, videotexto, publicaciones electrónicas, video, imagen, audio y los multimedios, 2) los datos de índole financiera y bursátil que apoyan el comercio internacional, y 3) la información gubernamental y no gubernamental (Sánchez Vanderkast, 2002: 58). Estos contenidos son, sin vía de duda, contenidos de las RS.

El reto está en interpretar estos flujos para que los servicios de información por vía de las RS ofrezcan indicadores que demuestren cómo impactan en los objetivos de la organización. 
Sánchez Vanderkast (2002: 60) nos presenta la clásica ecuación de Shannon cuando el tema se refiere a la evaluación de la eficiencia de la información. Con base en ello, la métrica de la eficiencia de la información es una relación que debe tomar en cuenta la aplicación de la información con base en los costos y el incremento de la comunicación, lo que es dado por la fórmula

$$
\operatorname{In}=\frac{\mathrm{K} \ln }{p}
$$

donde In representa la información, $\mathrm{K}$ es la constante, In es el logaritmo natural con base en la entropía y $p$ la probabilidad. Cuanto menor la entropía, menor la incertidumbre y mayor la eficiencia de la información. Aplicado a los sistemas humanos como los representados por las RS, la entropía es muy alta, especialmente por la dinámica de los seguidores.

Además de las características y las configuraciones de los dispositivos que acceden a las RS (e.g.: hacerlo desde un smartphone implica capacidades relacionadas a la conexión web, procesamiento, memoria, ubicación geográfica del usuario, interoperabilidad entre otras aplicaciones y servicios autorizados en el dispositivo del usuario, etc.), el valor de la información en el ciberespacio sigue dependiendo de contexto, de quién la produjo, para qué la produjo, y el grado de su impacto se puede identificar por las interacciones generadas en el grupo de usuarios, en el atendimiento a la oferta de productos/servicios/información, a muchas situaciones que determinan la manera con la cual uno se relaciona en una RS. Un estudiante universitario posiblemente se va asociar a la RS de su organización y eso no implica que las informaciones ahí le causen cambios de comportamiento o actitudes relacionadas a lo que ofrece la Universidad por ese medio.

La cantidad de información contenida en un mensaje, más allá del número de dígitos binarios (bits) necesarios para codificarla, implica las pérdidas, los ruidos, las distorsiones, las distracciones, las impresiones de los receptores, y eso vuelve muy alta la entropía de la información de una RS, ya que los niveles de interacción y respuesta de sus seguidores dependen de estos grados de influencia en sus aparatos, dispositivos, intereses personales, objetivos con la RS, comportamiento, entre otros.

Por eso, y teniendo en cuenta la interpretación de Weaver sobre la propuesta de Shannon, Sánchez Vanderkast (2002: 60) señala la consideración 
de los tres niveles de posibles problemas para la eficacia de la información con base en la entropía de los sistemas humanos, que son los de perspectiva técnica, la semántica, y la psico-conductual, así como las propuestas para superarlos (Tabla 1).

\begin{tabular}{|c|l|l|}
\hline Problemas & \multicolumn{1}{|c|}{ Descripción } & \multicolumn{1}{c|}{ Superación } \\
\hline Técnica & $\begin{array}{l}\text { Afectan la medida de la precisión de los } \\
\text { mensajes transmitidos y su recepción } \\
\text { por parte del destinatario. }\end{array}$ & La infraestructura adecuada. \\
\hline Semántica & $\begin{array}{l}\text { Con base en el modelo E-M-R, incurre } \\
\text { en el cuestionamiento sobre qué tan } \\
\text { precisos son los vocabularios utilizados } \\
\text { para describir algo o una cosa. }\end{array}$ & La selección de una fuente ideal. \\
\hline Psico-conductual & $\begin{array}{l}\text { Algo que se verá reflejado en la toma } \\
\text { de decisiones y la transformación de } \\
\text { conductas de los sujetos. }\end{array}$ & $\begin{array}{l}\text { El emisor: insumo, mensajes, preguntas. } \\
\text { El receptor: salida mensaje, problema } \\
\text { resuelto/toma de decisiones. }\end{array}$ \\
\hline
\end{tabular}

Tabla 1. Los tres niveles de posibles problemas para la eficacia de la información Fuente: adaptado de Sánchez Vanderkast (2002: 60-62)

En la búsqueda de indicadores que consideren tanto la eficiencia de la información como los mencionados niveles de posibles problemas para la eficacia de la información y la entropía de las RS, encontramos la métrica de los Key Performance Indicators (KPI). Los KPI son presentados como las principales métricas para evaluar los procesos de gestión. En función de los distintos objetivos en las RS, las organizaciones tendrán también distintos objetivos de acuerdo a su sistema gerencial y social, es decir, en función de aquello que quieran obtener con la información de las RS. En el caso de las RS de las entidades de la UNAM, queda claro que el objetivo de la Universidad es enriquecer la interacción y colaboración con la comunidad para que se logre una mayor visibilidad y apreciación del quehacer universitario (UNAM, 2014:3).

Los KPI responden por parte de estos indicadores, especialmente porque ellos se reportan a las características generales de las organizaciones. Fontalba (2018), consultora de Planeta Ttandem, presenta tres objetivos generales de las organizaciones en materia de uso de las RS, los cuales son métricas de los KPI para las RS (Figura 1). 


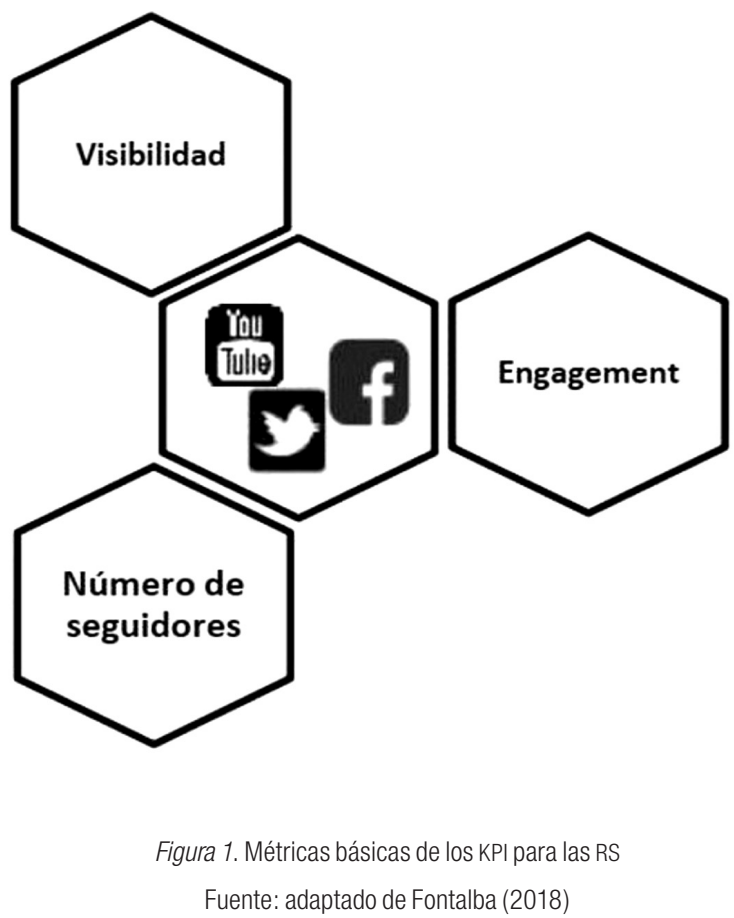

La visibilidad se refiere a dos indicadores: 1) de alcance, que es el número de personas que ha visto una publicación, y 2) de impresiones, que es el número de veces que las publicaciones fueron vistas. Un mismo usuario que ve varias veces una publicación posibilitará el registro de varias impresiones; sin embargo, el alcance de la publicación será sólo 1 por tratarse del mismo usuario. Para superar el sesgo y considerar el factor como un indicador clave, Mejía Llano (2019) propone la siguiente fórmula:

$$
\mathrm{U}=\frac{\mathrm{D}_{(\mathrm{U})}}{\mathrm{P}}
$$

donde $\mathrm{U}$ es el número de usuarios alcanzados promedio por publicación; $\mathrm{D}$ representa el número de usuarios alcanzados últimos 28 días, y $\mathrm{P}$ es el total publicaciones en el periodo de 28 días.

El número de seguidores, también llamado de tamaño de comunidad (Mejía Llano, 2019), se refiere al número total de seguidores de la RS (S) y 
permite identificar su crecimiento. El S es un número absoluto, lo cual se lee directamente en el perfil de la RS y para considerarlo como un KPI es importante tener en cuenta el día y la hora de su obtención.

El engagement se refiere a la generación de relación con el público (E). Según Fontalba (2018), cuanto mayor sea E, mayor la audiencia y la posibilidad de decisión del usuario por el producto o servicio. Se puede calcularlo en cada publicación realizada, en cada RS o en un conjunto de RS con la fórmula

$$
\mathrm{E}=\frac{\mathrm{I}_{(100)}}{\mathrm{U}}
$$

donde I es el total de interacciones. Son considerados como I la suma del número de likes con el número de shares y el número de comentarios. Eso es análogo a la consideración que hacen los investigadores en la exploración inicial de un tema: por lo general, se eligen los libros y artículos más conocidos, mejores evaluados por pares, y más citados para establecerse una referencia de lo que se va a leer. Una publicación en una RS que haya sido vista por 100 personas $(\mathrm{U}=100)$ y haya generado 25 likes, dos shares y tres comentarios ( $\mathrm{I}=30)$, tendrá un engagement de $30 \%(\mathrm{E}=30 \%)$.

Estas métricas básicas de los KPI para las RS pueden ser aplicadas a la totalidad de las organizaciones. En el mundo corporativo aún son considerados los KPI Click. Through Rate (CTR), Cost per Lead (CPL), la Conversión y el Return of Investment (ROI) (Mejía Llano, 2019). Como un segundo movimiento en esa proposición de indicadores de eficiencia de las RS de organizaciones universitarias, se hace la adaptación de los indicadores CTR y Conversión por entenderlos como aplicables a las instituciones públicas, como es el caso de la UNAM, ya que los otros presumen retorno financiero.

El KPI CTR indica el número de clics en cada publicación de una RS. Mejía Llano (2019) sugiere utilizar la herramienta Bitly.com, ya tradicionalmente conocida para identificar el número de clics en cada publicación. El factor de impacto se mide con la siguiente ecuación, donde $\mathrm{C}$ es el número de clics en los últimos 28 días:

$$
\mathrm{CTR}=\frac{\mathrm{C}_{(100)}}{\mathrm{U}}
$$


Desde un punto de vista corporativo, por efecto se debe esperar un mayor conocimiento de la marca así como un incremento en los ingresos de la corporación por mayor comercialización de sus productos/servicios. Para una organización universitaria pública, que no tiene la finalidad de generar ingreso financiero, ese retorno debe ser mensurado de otra manera.

Un primer retorno consiste en considerarse el KPI Conversión. Vilma Núñez (2018) define en lenguaje corporativo ese KPI como el porcentaje de usuarios que han pasado de fans a clientes e indica la fórmula siguiente para obtenerlo:

$$
\mathrm{PC}=\frac{\mathrm{S}_{(100)}}{\mathrm{W}}
$$

donde PC es el porcentaje de conversión y W indica el total de visitantes al sitio web desde las RS en el mismo periodo. Sin embargo, la simple visita al sitio web de la organización desde las RS no convierte un fan en un cliente o, mejor dicho, este indicador determina precisamente el aporte que está haciendo la RS en el sitio web, pero no garantiza que el seguidor se decida sobre la adquisición del producto/servicio de la organización. Una vez más, desde un punto de vista corporativo, el efecto impacta en el incremento de los ingresos de la empresa, pero para una organización pública es necesaria una mirada al detalle del flujo de la información en sus RS.

\section{LA PROPOSICIÓN DE KPI BAJO EL FLUJO DE LA INFORMACIÓN DE UNA ORGANIZACIÓN UNIVERSITARIA}

Con el reto bibliotecológico/de las Ciencias de la Información de interpretar los flujos de la información a través de las RS para ofrecer indicadores que demuestren cómo impactan en los objetivos de una organización universitaria, las primeras preguntas consideran la institucionalidad de la RS: ¿es necesario que haya agentes para que se dé el flujo de la información? ¿Quiénes son estos agentes? ¿Cómo se explican las propiedades del flujo informativo?

El sistema social del IIBI/UNAM tiene como soporte para el flujo de la información su público universitario de estudiantes, docentes, investigadores y técnicos internos y externos a la institución. Son ellos quienes producen y a quienes se dirige la información del quehacer de la entidad/Universidad. Sin embargo, la gestión de la información de la RS constituye tarea de agentes específicos de la entidad/Universidad. 
Ese debe ser, por lo tanto, la premisa básica de una RS de organización universitaria: el flujo de la información empieza en el momento en que se elige difundir ciertas informaciones. En ese artículo no se discute la política de información del IIBI/UNAM, pero sí la acción de poner la información en las RS, tarea de los gestores de la institución. En el caso del IIBI, la gestión de sus RS está a cargo del sector de Difusión y Educación Continua: a través de sus Técnicos Académicos, toda la información de los trabajos, eventos y actividades del Instituto son publicadas en las RS y de ahí acompañadas en su proceso de impacto. La propuesta para evaluar ese proceso ha sido diseñada en este artículo.

En la construcción de los KPI bajo ese flujo de la información, la premisa que sigue es clasificar los tipos de información que son publicadas por el sector de Difusión y Educación Continua. Del análisis se obtienen tres tipos de información de las RS IIBI (Tabla 2).

\begin{tabular}{|c|l|l|}
\hline \multicolumn{1}{|c|}{ Clase } & \multicolumn{1}{|c|}{ Definición } & \multicolumn{1}{c|}{ Ejemplos } \\
\hline Institucionales & $\begin{array}{l}\text { Emitidas por la alta gestión de la Universidad, } \\
\text { la Rectoría, Secretarías, Direcciones. }\end{array}$ & $\begin{array}{l}\text { Boletín, Informes periódicos, } \\
\text { campañas, noticias de carácter } \\
\text { institucional. }\end{array}$ \\
\hline Notas informativas & $\begin{array}{l}\text { Emitidas por la alta gestión de la Universidad } \\
\text { ypor la entidad. }\end{array}$ & $\begin{array}{l}\text { Información de respuesta } \\
\text { a situaciones internas y externas } \\
\text { a la Universidad/entidad, } \\
\text { instrucciones a la comunidad. }\end{array}$ \\
\hline Eventos & Emitidas por la entidad. & $\begin{array}{l}\text { Difusión de talleres, cursos, } \\
\text { congresos, libros, participación } \\
\text { de la entidad en organismos } \\
\text { nacionales e internacionales. }\end{array}$ \\
\hline
\end{tabular}

Tabla 2. Tipos de información de las RS del IIBI/UNAM

Las respuestas de la comunidad interna y externa de la UNAM a cada uno de estos tipos de información son distintas, así como la forma de evaluar su impacto. De hecho, podemos aplicar a los tres grupos los indicadores de visibilidad, engagement y CTR; la Conversión interesa al tipo de información "eventos", los cuales pueden ser mensurados con la participación de los seguidores (fans) en las actividades de la entidad como su público (clientes). Así, se propone para el indicador Conversión el siguiente KPI para la organización universitaria:

$$
\mathrm{PC}=\frac{\mathrm{A}_{(100)}}{\mathrm{U}}
$$


donde A representa el número de personas que han participado de la actividad (asistentes) por obtener información desde las RS. Hay que clasificar también el tipo de participación en la actividad, si es presencial o en línea. Eso porque la RS YouTube tiene un objetivo diferenciado para las actividades de la organización: a través de ella se puede tanto acceder a la publicidad de un evento, nota informativa, información institucional o memoria de un evento, como también participar en ello a través de su transmisión en vivo (livestream). Así, ese KPI puede ser total (presencial + en línea) o por modalidades (presencial y en línea).

\section{Conclusión}

Ese artículo concluye planteando un diseño de indicadores de eficiencia para las RS de una organización universitaria (Figura 2).

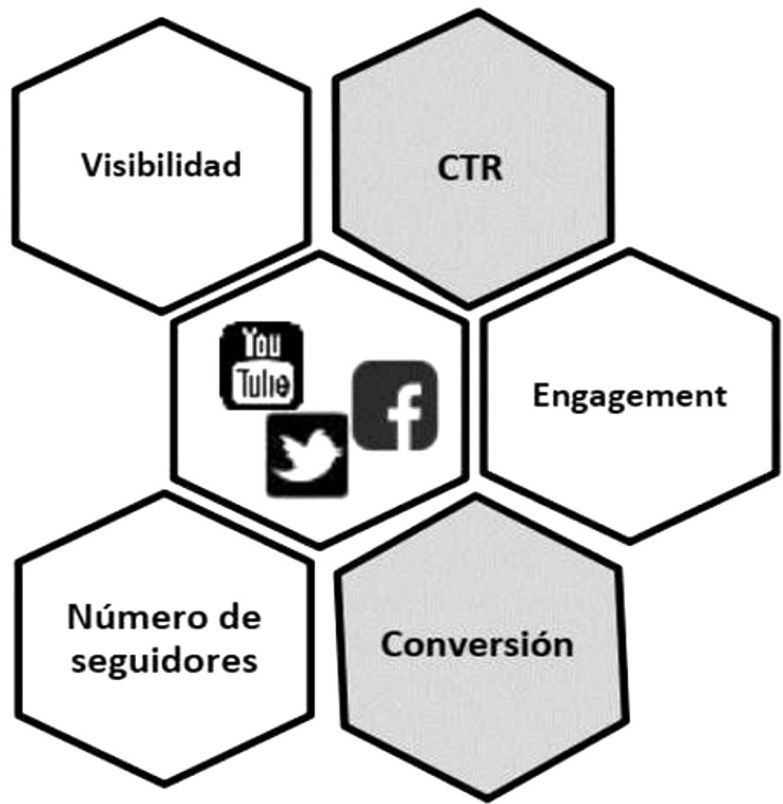

Figura 2. Métricas de los KPI para las RS de organizaciones universitarias 
Mientras que el KPI Conversión es una adaptación dada por la ecuación

$$
P C=\frac{A_{(100)}}{U}
$$

Eso porque, sin presumir retorno financiero, las organizaciones universitarias públicas como la UNAM impactan sus actividades en otras bases, siendo la principal el nivel de participación de su público $(\mathrm{A})$ interno y externo en el consumo de los productos y servicios de la institución.

Además, ese estudio ha aportado algunas bases teórico-prácticas para las investigaciones futuras que consideren el creciente uso de las RS por las organizaciones universitarias en sus procesos de gestión, ofreciendo las métricas hacia una metodología de evaluación de RS que tiene en cuenta sus aspectos socio-técnicos, el flujo de la información de la organización y la dinámica que asume la información en el contexto de la RS generales.

\section{Agradecimientos}

Al Gobierno de México, que a través de la Agencia Mexicana de Cooperación Internacional para el Desarrollo (AMEXCID) de la Secretaría de Relaciones Exteriores (SRE) ha patrocinado el proyecto de investigación "El flujo de información a través de las redes sociales".

A la Universidad Nacional Autónoma de México (UNAM), que a través del Instituto de Investigaciones Bibliotecológicas y de la Información (IIBI)

ha ofrecido todas las condiciones técnicas y científicas para la realización de ese estudio. A la Dirección del IIBI, la Secretaría Académica y la Secretaría Administrativa por todo el apoyo y soporte técnico en el proyecto. Al personal del Departamento de Cómputo y del Departamento de Difusión y Educación Continua. Al Seminario de Investigación Metadatos. 


\section{REFERENCIAS}

AIMX (Asociación de Internet MX). 2019. 15Estudio sobre los Hábitos de los Usuarios de Internet en México 2019. Ciudad de México: Asociación de Internet MX. Fecha de consulta: 30 de noviembre de 2019.

https://www.asociaciondeinternet.mx/estudios/habitos-de-internet

Caldevilla Dominguez, David. 2010. "Las Redes Sociales. Tipología, uso y consumo de las redes 2.0 en la sociedad digital actual". Documentación de las Ciencias de la Información 33 (julio): 45-68.

https://revistas.ucm.es/index.php/DCIN/article/view/DCIN1010110045A/18656

Celaya, Javier. 2008. La empresa en la Web 2.0. El impacto de las redes sociales y las nuevas formas de comunicación online en la estrategia empresarial, Barcelona: Gestión 2000.

Contreras Orozco, Leticia. 2017. "Las redes sociales digitales como factor de proximidad entre la sociedad y los gobiernos subnacionales en México”. Revista Iberoamericana de las Ciencias Sociales y Humanísticas 6 (12) (julio/diciembre): 225-249. https://doi.org/10.23913/ricsh.v6i12.131

Devlin, Keith. 1991. Logic and Information, Cambridge: Cambridge University Press.

Emery, Fred Edmund y Eric Lansdown Trist. 1960. Socio-technical Systems. Management Sciences Models and Techniques, London: Pergamon Press.

Fontalba, Txaro. 2018. "KPIs Redes Sociales. Los KPIs más importantes en Redes Sociales según los objetivos”. Planeta Ttandem (blog). Fecha de consulta: 15 de enero de 2020. https://www.ttandem.com/blog/los-kpis-mas-importantes-en-redes-sociales-segun-los-objetivos

Hütt Herrera, Harold. 2012. "Las redes sociales: una nueva herramienta de difusión”. Reflexiones 91 (2): 121-128. http://www.redalyc.org/articulo.oa?id=72923962008

Kemp, Simon. 2019. Digital 2019: essential insights into how people around the world use the internet, mobile devices, social media, and e-commerce. Vancouver: Hootsuite. Fecha de consulta: 30 de noviembre de 2019. https://hootsuite.com/resources/digital-in-2019

Kingdon, Jhon Wells. 1973. Agendas, Alternatives and Public Policies, New York: Harper Collins.

Manrique Valenzuela, Katy y Miguel Domingo Gonzalez Álvarez. 2013. "Desarrollo de Sistemas Socio Técnicos en el área de Seguridad y Salud Ocupacional de una empresa de servicio". Trabajo presentado en el Congreso ALTEC 2013, Porto, Portugal, 23-31 de octubre. Fecha de consulta: 15 de enero de 2020. http://www.altec2013.org/programme_pdf/429.pdf

Mejía Llano, Juan Carlos. 2019. "Indicadores de Redes Sociales: principales KPIs y métricas de Social Media”, Juan Carlos Mejía Llano Blog (blog) Acceso el 15 de enero de 2020

https://www.juancmejia.com/redes-sociales/kpis-de-redes-sociales-guia-con-principales-metricas-e-indicadores-de-social-media

Núñez, Vilma. 2018. "Indicadores KPI: Qué son, cuántos tipos existen y recomendaciones”, VN Blog (blog). Fecha de consulta: 15 de enero de 2020. https://vilmanunez.com/indicadores-kpi 
Ríos Ortega, Jaime. 2012. Plan de Desarrollo Institucional. Instituto de Investigaciones Bibliotecológicas y de la Información 2012-2016. Ciudad de México: IIBI/UNAM. Fecha de consulta: 15 de enero de 2020. http://iibi.unam.mx/f/PDI_IBII_UNAM_2012_2016.pdf

Ríos Ortega, Jaime. 2014. $2^{\circ}$ Informe de Actividades 2013-2014 del Institucional Instituto de Investigaciones Bibliotecológicas y de la Información, Ciudad de México: IIBI/UNAM. Fecha de consulta: 25 de enero de 2020. http://iibi.unam.mx/f/2_informe_iibi_2013 2014.pdf

Sánchez Vanderkast, Egbert John. 2002. "Los flujos de información: algunas reflexiones". Investigación Bibliotecológica 16 (32) (enero/junio): 55-70. http://dx.doi.org/10.22201/iibi.0187358xp.2002.32.3995

Terra, Ana Lucia y Ariel Alejandro Rodríguez García. 2013. “Descifrando la reestructuración técnica del profesional de la información con las argumentaciones de la economía global". Trabajo presentado en el VI Encontro Ibérico EDICIC, Porto, Portugal, 4-6 de noviembre. Fecha de consulta: 03 de febrero de 2020. http://eprints.rclis.org/22841

Torres Vargas, Georgina Araceli, Egbert John Sánchez Vanderkast, Ariel Alejandro Rodríguez García y Jenny Teresita Guerra González. 2015. "The Blended Librarian and the Disruptive Technological Innovation in the Digital World". Open Access Library Journal 2 (1764): 1-9. https://www.scirp.org/journal/PaperInformation.aspx?PaperID=68542\&

Trist, Eric Lansdown y Ken Bamforth. 1951. "Some social and psychological consequences of the Longwall Method of coal-getting: an examination of the psychological situation and defences of a work group in relation to the social structure and technological content of the work system". Human relations 4 (1) (febrero): 3-38. https://doi.org/10.1177/001872675100400101

Triviños, Augusto Nivaldo Silva. 1987. Introdução à pesquisa em ciências sociais: a pesquisa qualitativa em educação. São Paulo: Atlas.

UNAM (Universidad Nacional Autónoma de México). 2012. Plan de Desarrollo Institucional 2011-2015. Ciudad de México: UNAM. Acceso 28 de noviembre de 2019. http://www.planeacion.unam.mx/consulta/Plan_desarrollo.pdf

UNAM. 2014. Instructivo de uso para Redes Sociales Institucionales de la UNAM, Ciudad de México: UNAM/DGCS. Dirección General de Comunicación Social, Comité de Comunicación de la Dirección General de Comunicación Social. Fecha de consulta: 28 de noviembre de 2019.

https://www.visibilidadweb.unam.mx/normateca/normaunam/Lineamientosredessociales.pdf

UNAM. 2017. Plan de Desarrollo Institucional 2015-2019, Ciudad de México: UNAM. Fecha de consulta: 28 de noviembre de 2019. http://www.rector.unam.mx/doctos/PDI-2015-2019.pdf

UNAM. 2020. Visibilidad web UNAM. Dirección General de Comunicación Social, Comité de Comunicación de la Dirección General de Comunicación Social. Fecha de consulta: 28 de noviembre de 2019. https://www.visibilidadweb.unam.mx/redes.html

Veletsianos, George. 2011. "Higher education scholars' participation and practices on Twitter”. Journal of Computer Assisted Learning 28 (4): 1-14. https://doi.org/10.1111/j.1365-2729.2011.00449.x 
Para citar este texto:

Brandão, Marco. 2021. "Una revisión sociotécnica de las redes sociales de una organización universitaria". Investigación Bibliotecológica: archivonomia, bibliotecología e información 35 (86): 161-184.

http://dx.doi.org/10.22201/iibi.24488321xe.2021.86.58281 